\title{
Comparative Evaluation of the Inhibitory Effect of Some Essential Oils with Antibiotics against Pseudomonas aeruginosa
}

\author{
Lobna El-Hosseiny, ${ }^{1}$ Moustafa El-Shenawy, ${ }^{2}$ Medhat Haroun, ${ }^{3}$ and Fadhil Abdullah ${ }^{3}$ \\ ${ }^{1}$ Department of Environmental Studies, Institute of Graduate Studies and Research, Alexandria University, Egypt \\ ${ }^{2}$ Department of Food Microbiology, National Research Center, Dokki, Cairo, Egypt \\ ${ }^{3}$ Department of Biotechnology, Institute of Graduate Studies and Research, Alexandria University, Egypt
}

Correspondence should be addressed to Lobna El-Hosseiny; lobnaelhosseiny@yahoo.com

Received 29 June 2014; Revised 24 September 2014; Accepted 24 September 2014; Published 1 October 2014

Academic Editor: Branka Bedenić

Copyright (C) 2014 Lobna El-Hosseiny et al. This is an open access article distributed under the Creative Commons Attribution License, which permits unrestricted use, distribution, and reproduction in any medium, provided the original work is properly cited.

Gas chromatography/mass spectroscopy analysis was performed to identify the chemical components of three extracted essential oils including thyme, marjoram, and sage. The antibacterial activity of the extracted essential oils against Pseudomonas aeruginosa (ATCC 9027) was investigated using disc diffusion assay, either alone or in combination with standard antibiotics (piperacillin, cefepime, meropenem, gentamicin, and norfloxacin). Results showed that the studied oils exhibited a variety of activities against the tested bacterium. Thyme oil was the most active followed by marjoram oil, whereas sage displayed no activity towards the tested organism. Thyme oil enhanced the antibacterial activity of cell wall targeting antibiotics (piperacillin, cefepime, and meropenem) by more than twofold. Marjoram oil potentiated the activity of all the tested antibiotics except norfloxacin. Sage, despite its inactivity against pseudomonas, synergistically enhanced the activity of piperacillin, meropenem, and gentamicin. Thyme essential oil, containing thymol as a major component (33.6\%), exhibited higher activity alone or in combination with antibiotics than marjoram which contained alcoholic terpenes or sage essential oil that contained 1,8-cineole as its major component (29\%). The investigated oils, as natural bioactive agents, may be used to enhance the activity of antibiotics towards pseudomonas.

\section{Introduction}

Among all of the bacterial resistance problems, Gram-negative pathogens are particularly worrisome, because they are becoming resistant to nearly all drugs that would be considered for treatment. The most serious Gram-negative infections are health-care associated and the most common pathogens are Enterobacteriaceae, Pseudomonas, and Acinetobacter [1]. Pseudomonas aeruginosa is one of the leading causes of nosocomial infections and is associated with high mortality. Infections caused by Pseudomonas are difficult to treat as the repertoire of useful antipseudomonal agents is limited. Moreover, Pseudomonas exhibits remarkable abilities to acquire resistance to these agents [2].

Historically plants have enjoyed a tradition of use for their flavor enhancement characteristics and for their medicinal properties. A growing body of evidence points to the antimicrobial potentials of plant extracts in an era of prevalence of antibiotics resistance. It is reported that plant derived antimicrobials have higher minimum inhibitory concentrations (MICs) than bacterial and fungal produced antibiotics $[3,4]$. This provides the rationale for research into the potential outcome of plant derived essential oils on the in vitro activity of antibiotics.

In view of the dearth, in the near future, of new options against resistant $P$. aeruginosa strains, plants provide a potential source for therapeutically useful compounds especially from the perspective of their potentials in combination with antimicrobial chemotherapy. Accordingly, the current study investigated the antimicrobial enhancing effect of three different essential oils, extracted from some medicinal plants, against the bacterium Pseudomonas aeruginosa, when combined with conventional antibiotics most commonly used in the treatment of such pathogen. 


\section{Material and Methods}

2.1. Plant Material and Essential Oil Extraction. Dried leaves of thyme, marjoram, and sage were obtained from the local market, Alexandria, Egypt. The dried plant material was subjected to hydrodistillation by Clevenger type apparatus for $4 \mathrm{~h}$. The oils were collected, dried over anhydrous sodium sulphate, and stored in sealed vials at $4^{\circ} \mathrm{C}$ until analysed.

2.2. Organism and Culture Conditions. Pseudomonas aeruginosa ATCC 9027 was obtained from the stock culture collection of the Department of Food Microbiology at the National Research Center, Dokki, Cairo. The strain was routinely maintained in tryptone soya agar (Oxoid) and stored at $4^{\circ} \mathrm{C}$. The culture was activated in brain heart infusion (Oxoid) followed by Müeller Hinton broth (MHB, Oxoid) and incubated overnight at $35^{\circ} \mathrm{C}$ for $18 \mathrm{hrs}$ for subsequent antibacterial assay.

2.3. Chemical Analysis of Essential Oils. The chemical composition of the extracted essential oils was analysed using a thermo scientific GC/MS version (5) 2009 system with TG$5 \mathrm{MS}$ column $(30 \mathrm{~m} \times 0.32 \mathrm{~mm}$ ID). Five $\mu \mathrm{L}$ essential oil was diluted to $1 \mathrm{~mL}$ with dichloromethane; then $2 \mu \mathrm{L}$ was injected on splitless mode for $1 \mathrm{~min}$, followed by a split flow with ratio $1: 10$. GC oven temperature was programmed from $60^{\circ} \mathrm{C}$ to $280^{\circ} \mathrm{C}$ at $3^{\circ} \mathrm{C} / \mathrm{min}$ using helium as a carrier gas. Both the interface and injection temperatures were adjusted at $250^{\circ} \mathrm{C}$. The ionization voltage was $70 \mathrm{eV}$ with a mass range between 40 and $800 \mathrm{~m} / \mathrm{z}$. Identification of the oil constituents was done on the basis of retention index (RI, determined with reference to homologous series of $n$-alkanes C8-C25, under identical experimental condition) and MS library search (NIST and WILEY) and by comparison with MS literature data [5]. The relative amounts of individual components were calculated based on GC peak area.

2.4. Antibacterial Assay. Müeller Hinton agar plates (MHA, Oxoid) were surface-inoculated with $10^{7} \mathrm{CFU} / \mathrm{mL}$ of the overnight $\mathrm{MHB}$ culture. For screening the activity of essential oils, sterile paper discs (6 $\mathrm{mm}$ in diameter) saturated with $2 \mu \mathrm{L}$ of the corresponding essential oil were placed on the surface of MHA that contained $0.5 \%$ Tween 20 (Sigma) to enhance the diffusion of essential oils. The antibiotic sensitivity pattern of $P$. aeruginosa strain was studied by Kirby Bauer's disc diffusion method using commercially available antibiotic discs (Oxoid), piperacillin $(100 \mu \mathrm{g})$, cefepime $(30 \mu \mathrm{g})$, meropenem $(10 \mu \mathrm{g})$, gentamicin $(10 \mu \mathrm{g})$, and norfloxacin $(10 \mu \mathrm{g})$, as per the Clinical and Laboratory Standards Institute [6] standards. Meanwhile, assay of the activity of the combination of essential oils and the antibiotics was carried out by impregnating the standard antibiotic discs with $2 \mu \mathrm{L}$ of the essential oil. The plates, after $2 \mathrm{hrs}$ of maintenance at $4^{\circ} \mathrm{C}$, were incubated at $37^{\circ} \mathrm{C}$ for $18-24 \mathrm{hrs}$ and the diameter of the resulting zones of inhibition was measured in millimeter.

2.5. Statistical Analysis. Results obtained were subjected to statistical analysis using one-way analysis of variance (SPSS 17). All data were the mean of three experiments.

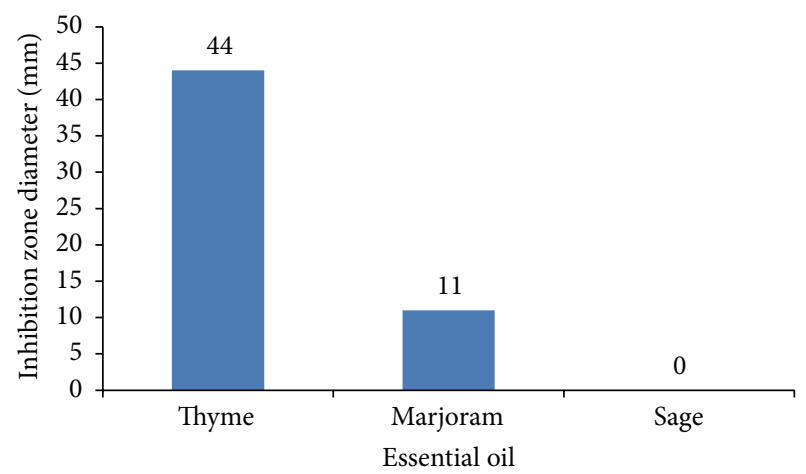

FIgURe 1: Activity of the examined essential oils against Pseudomonas aeruginosa.

\section{Results and Discussion}

Essential oils currently investigated displayed variable activities towards the tested Pseudomonas aeruginosa strain. Thyme essential oil exhibited the highest activity as conveyed by its highest inhibition zone. Meanwhile marjoram displayed a moderate activity whereas sage oil exhibited no effect against the test pathogen (Figure 1). In a study of the antibacterial activity of Thymus vulgaris essential oil against multidrug resistant $P$. aeruginosa strains, using the agar diffusion test, the oil exhibited a strong antipseudomonal activity with an inhibition zone diameter of 28-36 mm [7]. Meanwhile, sage oil was reported to demonstrate no effect against the Gramnegative pathogen $P$. aeruginosa [8]. Furthermore, a study of the antibacterial activity of sage oil against a group of urinary tract pathogens comprising $P$. aeruginosa, Klebsiella, E. coli, Proteus mirabilis, and Morganella showed a 100\% efficiency against Klebsiella, 96\% efficiency against E. coli, 83\% efficiency against Proteus mirabilis, 75\% efficiency against Morganella morganii, and $0 \%$ efficiency against Pseudomonas aeruginosa [9]. In a study of the in vitro antimicrobial activity of essential oils from Mediterranean Lamiaceae plants against food borne pathogens and spoilage bacteria, it has been reported that, among Lamiaceae plants investigated, thyme and marjoram oils inhibited the pseudomonal strain tested with varying degrees where thyme recorded lower MIC values compared to marjoram [10]. The results of the current investigation are in agreement with previous studies in the context of order of susceptibility of Pseudomonas to the three investigated essential oils.

Based on CLSI susceptibility interpretation criteria, the currently investigated Pseudomonas aeruginosa strain presented a resistance profile comprising resistance towards piperacillin but not the aminoglycoside "gentamicin," the fluoroquinolone "norfloxacin," the carbapenem "meropenem," or the 4th generation cephalosporin "cefepime" (Table 1). Pseudomonas aeruginosa is reported to be naturally resistant to several antibiotics owing to its relatively impermeable membrane, constitutively expressed and inducible efflux systems, and a chromosomally encoded inducible $\beta$-lactamase [11]. Pseudomonas was classified into several phenotypes according to its resistance patterns with the susceptible phenotype 
TABLE 1: Susceptibility pattern of Pseudomonas aeruginosa to tested antibiotics.

\begin{tabular}{lcccccc}
\hline Antibiotic & Disc content & $\begin{array}{c}\text { Inhibition zone } \\
\text { diameter }(\mathrm{mm})\end{array}$ & $\begin{array}{c}\text { Zone diameter breakpoints according to CLSI* guidelines } \\
\mathrm{S}\end{array}$ & $\begin{array}{c}\text { Sensitivity } \\
\text { pattern }\end{array}$ \\
\hline Piperacillin & $100 \mu \mathrm{g}$ & 15 & $\geq 18$ & - & $\leq 17$ & Resistant \\
Cefepime & $30 \mu \mathrm{g}$ & 30 & $\geq 18$ & $15-17$ & $\leq 14$ & Susceptible \\
Meropenem & $10 \mu \mathrm{g}$ & 34 & $\geq 16$ & $14-15$ & $\leq 13$ & $\leq 12$ \\
Gentamicin & $10 \mu \mathrm{g}$ & 29 & $\geq 17$ & $13-16$ & $\leq 12$ & Susceptible \\
Norfloxacin & $10 \mu \mathrm{g}$ & 31 & & Susceptible \\
\hline
\end{tabular}

${ }^{*}$ CLSI: Clinical and Laboratory Standards Institute.

TABLE 2: Inhibition zone diameter of the tested antibiotics alone and in combination with essential oils.

\begin{tabular}{lcccc}
\hline Antibiotic & $\begin{array}{c}\text { Inhibition zone } \\
\text { diameter }(\mathrm{mm})\end{array}$ & $\begin{array}{c}\text { Antibiotic + thyme essential oil } \\
(\% \text { enhancement })\end{array}$ & $\begin{array}{c}\text { Combination of antibiotics and essential oils } \\
\text { essential oil } \\
(\% \text { enhancement })\end{array}$ & $\begin{array}{c}\text { Antibiotic + sage essential oil } \\
(\% \text { enhancement })\end{array}$ \\
\hline Piperacillin & $17^{\mathrm{d}}$ & $42^{\mathrm{a}}(147 \%)$ & $24^{\mathrm{b}}(41.2 \%)$ & $22^{\mathrm{c}}(29.4 \%)$ \\
Cefepime & $30^{\mathrm{c}}$ & $46^{\mathrm{a}}(53.3 \%)$ & $33^{\mathrm{b}}(10 \%)$ & $30^{\mathrm{c}}(-0.03 \%)$ \\
Meropenem & $34^{\mathrm{c}}$ & $52^{\mathrm{a}}(52.9 \%)$ & $41^{\mathrm{b}}(20.5 \%)$ & $42^{\mathrm{b}}(23.5 \%)$ \\
Gentamicin & $29^{\mathrm{d}}$ & $40^{\mathrm{a}}(37.9 \%)$ & $38^{\mathrm{b}}(31.03 \%)$ & $33^{\mathrm{c}}(13.7 \%)$ \\
Norfloxacin & $31^{\mathrm{b}}$ & $43^{\mathrm{a}}(38.7 \%)$ & $30^{\mathrm{b}}(-0.03 \%)$ & $26^{\mathrm{c}}(-0.16 \%)$ \\
\hline
\end{tabular}

${ }^{\text {abcd }}$ Mean values within a row not sharing common superscript letters are significantly different, $P<0.05$.

being sensitive to carboxypenicillins (carbenicillin and ticarcillin), ureidopenicillins (azlocillin and piperacillin), some third generation cephalosporins (ceftazidime and cefoperazone), all the fourth generation cephalosporins, the monobactam "aztreonam," and the carbapenems (imipenem and meropenem). The resistant phenotypes display variable resistance patterns, the first phenotype being resistant to non- $\beta$ lactam antibiotics as quinolones, trimethoprim, tetracycline, and chloramphenicol besides being resistant to most of the $\beta$-lactams including meropenem but not imipenem. The second phenotype displays resistance to all beta-lactams except cephems (cefepime and cefpirome) and carbapenems. In the third phenotype resistance to penicillins, in particular piperacillin and azlocillin and ticarcillin, is affected more than resistance to cephalosporins $[12,13]$. The resistance pattern displayed by the currently investigated Pseudomonas strain (ATCC 9027) is in general agreement with the second phenotype in terms of displaying resistance to the betalactam piperacillin but not the cephem "cefepime" or the carbapenem "meropenem."

Essential oils are highly complex mixtures of volatile compounds including terpene hydrocarbons and their oxygenated derivatives constituting alcohols, esters, aldehydes, ketones, phenols, and ethers [14]. Although essential oils are complex mixtures, their activity can be accounted for in terms of their major terpene or terpenoid components. It is reported that most of the antimicrobial activity in essential oils is found in the oxygenated terpene components. Moreover, it has been demonstrated that whole essential oils usually have higher antibacterial activity than the mixtures of their major components [15].
GC/MS analysis of thyme essential oil, in the current investigation, revealed that the phenolic terpene thymol was the major component constituting $33.6 \%$ of the oil composition; in marjoram oil the alcoholic terpenes including 4 -terpineol, $\alpha$-terpineol, cis- $\beta$-terpineol, and linalool were the major components comprising $21.3 \%, 5.31 \%, 5.24 \%$, and $2.53 \%$, respectively. The terpenoid ether, 1,8 -cineole, was the major component in sage oil representing $29 \%$ of the total essential oil composition.

In studies investigating the antimicrobial activity of phenolic and alcoholic terpenoids in essential oils, it has been reported that essential oils containing aldehydes or phenols as major components demonstrated the highest antibacterial activity followed by essential oils containing terpene alcohols [15]. These findings are in accordance with the results recorded in the current investigation (Figure 1 and Table 2), where thyme essential oil containing terpenoid phenols exhibited a higher antibacterial activity than essential oils containing alcoholic terpenoids (marjoram oil) or terpenoid ethers (sage oil).

The phenolic terpenoids, thymol and eugenol, were amongst the terpenoids that attracted the attention of researchers. Their antimicrobial action was elucidated to be related to the cell membrane of bacteria. The primary mode of action of thymol involves outer and inner membrane disruption and interaction with membrane proteins and intracellular targets [16]. Thymol has been reported to integrate at the polar head region of the lipid bilayer leading to alteration in membrane phospholipids and leakage of potassium ions and ATP $[17,18]$. In this regard, results in the current study indicated that thyme essential oil, with its high content of the phenolic 
monoterpene thymol, exhibited the highest antipseudomonal activity when used singly or jointly with the tested conventional antibiotics. The activity of these antibiotics was significantly enhanced by more than twofold especially those targeting the cell wall including piperacillin, cefepime, and meropenem (Table 2).

The chemical composition of marjoram essential oil investigated in the current study revealed that it contains alcoholic terpenes with various structural configurations, comprising $4-, \alpha$-, and $\beta$-terpineol which are cyclic aliphatic monoterpene alcohols in addition to linalool which is an acyclic terpene alcohol. These alcohols collectively constituted about one-third of the total essential oil composition. Links between oxygenated terpenes and antimicrobial activity have been investigated in several studies. In a study of the activity of $\alpha$-citral, linalool, geraniol, and citronellol present in lemon grass oil, it was found that these oxygenated terpenes were active against S. aureus, E. coli, and B. subtilis, whereas nonoxygenated terpene hydrocarbons as limonene exhibited no activity [19]. In another study, the oxygenated terpene alcohol 4-terpineol, abundant in tea tree oil, exhibited bactericidal properties and oils with high 4-terpineol contents demonstrated increased antimicrobial activities [20]. Moreover, despite the minor abundance of $\alpha$-terpineol and linalool in tea tree oil, they exhibited similar antimicrobial activities as 4-terpineol [21]. In the current study, linalool and $\alpha$-terpineol, although present in relatively small amounts compared to 4-terpineol, in marjoram essential oil, acted synergistically and contributed to the overall antipseudomonal activity. Accumulating evidence has demonstrated that oxygenated terpenes, particularly phenolic and alcoholic terpenoids, inhibit microbial oxygen uptake and oxidative phosphorylation. Meanwhile, it was suggested that the free $\mathrm{OH}$ group possessed by both alcohols and phenols is a key to their activity $[16,22]$. In corroboration with these findings, the current results indicated that both thyme and marjoram essential oils exhibited higher potentiating capacity when combined with piperacillin, cefepime, and gentamicin compared to sage essential oil in which its major components are devoid of the free $\mathrm{OH}$ group (Table 2).

It has been reported that plants either contain antimicrobials that can operate in synergy with antibiotics or possess compounds that have no intrinsic antibacterial activity but are able to sensitize the pathogen to an ineffective antibiotic [23]. In this context, although sage essential oil in the current study displayed no activity against the tested pathogen, it significantly potentiated the activity of three of the five cotested antibiotics, namely, piperacillin, meropenem, and gentamicin. In the consensus of plant derived antimicrobials and their structural diversity, it has been documented that most of these molecules have weak antibiotic activity, several orders of magnitude less than that of common antibiotics produced by bacteria and fungi. In spite of the fact that plant derived antibacterials are less potent, plants fight infections successfully. Hence, it becomes apparent that plants adopt a different paradigm, "synergy," to combat infections [24]. Furthermore, the likelihood that a pathogen could simultaneously develop resistance against more than one drug is low [25]. Hence, essential oils and their multiple components are candidate agents that are proposed to act on several targets, meanwhile reducing the probability of resistance development.

Conceivably, the investigated essential oils, with their complex composition, can provide a source for natural agents that may enhance the activity of some antibiotics against Pseudomonas aeruginosa. Still, issues like the mechanism of their antimicrobial action need to be addressed to underpin the complexity of synergistic potential between natural plant extracts and antibiotics against bacteria.

\section{Conflict of Interests}

The authors declare that there is no conflict of interests regarding the publication of this paper.

\section{References}

[1] Center for Disease Control (CDC), Antibiotic Resistance Threats in the United States, 2013, 2013.

[2] G. M. Rossolini and E. Mantengoli, “Treatment and control of severe infections caused by multiresistant Pseudomonas aeruginosa," Clinical Microbiology and Infection, vol. 11, no. s4, pp. 1732, 2005.

[3] B. Marquez, L. Neuville, N. J. Moreau et al., "Multidrug resistance reversal agent from Jatropha elliptica," Phytochemistry, vol. 66, no. 15, pp. 1804-1811, 2005.

[4] E. C. J. Smith, E. M. Williamson, N. Wareham, G. W. Kaatz, and S. Gibbons, "Antibacterials and modulators of bacterial resistance from the immature cones of Chamaecyparis lawsoniana," Phytochemistry, vol. 68, no. 2, pp. 210-217, 2007.

[5] R. P. Adams, Identification of Essential Oil Components by Gas Chromatography/Mass Spectrometry, Allured Publishing Corporation, Carol Stream, Ill, USA, 4th edition, 2007.

[6] Clinical and Laboratory Standards Institute (CLSI), "Performance standards for antimicrobial susceptibility testing; twentyfirst informational supplement," Tech. Rep. M100 -S21, Clinical and Laboratory Standards Institute, Wayne, Pa, USA, 2011.

[7] W. A. El-Shouny and S. Magaam, "Sensitivity of Multi-drug Resistant Pseudomonas aeruginosa isolated from surgical woundinfections to essential oils and plant Extracts," World Journal of medical Sciences, vol. 4, pp. 104-111, 2009.

[8] T. Bosnic, D. Softic, and J. G. Vasic, "Antimicrobial activity of some essential oils and major constituents of essential oils," Acta Medica Academica, vol. 35, pp. 19-22, 2006.

[9] R. Santos Pereira, T. C. Sumita, M. R. Furlan, A. O. Cardoso Jorge, and M. Ueno, "Antibacterial activity of essential oils on microorganisms isolated from urinary tract infection," Revista de Saude Publica, vol. 38, no. 2, pp. 326-328, 2004.

[10] R. Di Pasqua, V. de Feo, F. Villani, and G. Mauriello, "In vitro antimicrobial activity of essential oils from Mediterranean Apiaceae, Verbenaceae and Lamiaceae against foodborne pathogens and spoilage bacteria," Annals of Microbiology, vol. 55, no. 2, pp. 139-143, 2005.

[11] P. D. Lister, D. J. Wolter, and N. D. Hanson, "Antibacterialresistant Pseudomonas aeruginosa: clinical impact and complex regulation of chromosomally encoded resistance mechanisms," Clinical Microbiology Reviews, vol. 22, no. 4, pp. 582-610, 2009.

[12] J. C. Pechere and T. Kohler, "Patterns and modes of $\beta$-lactam resistance in Pseudomonas aeruginosa," Clinical Microbiology and Infection, vol. 5, no. 1, pp. S15-S18, 1999. 
[13] T. Strateva and D. Yordanov, "Pseudomonas aeruginosa: a phenomenon of bacterial resistance," Journal of Medical Microbiology, vol. 58, no. 9, pp. 1133-1148, 2009.

[14] A. Djilani and A. Dick, The Therapeutic Benefits of Essential Oils, Nutrition, Well-Being and Health, 2012.

[15] I. H. N. Bassolé and H. R. Juliani, "Essential oils in combination and their antimicrobial properties," Molecules, vol. 17, no. 4, pp. 3989-4006, 2012.

[16] M. Hyldgaard, T. Mygind, and R. L. Meyer, "Essential oils in food preservation: mode of action, synergies, and interactions with food matrix components," Frontiers in Microbiology, vol. 3 , article 12, 2012.

[17] R. di Pasqua, G. Mamone, P. Ferranti, D. Ercolini, and G. Mauriello, "Changes in the proteome of Salmonella enterica serovar Thompson as stress adaptation to sublethal concentrations of thymol," Proteomics, vol. 10, no. 5, pp. 1040-1049, 2010.

[18] J. Xu, F. Zhou, B.-P. Ji, R.-S. Pei, and N. Xu, “The antibacterial mechanism of carvacrol and thymol against Escherichia coli," Letters in Applied Microbiology, vol. 47, no. 3, pp. 174-179, 2008.

[19] G. O. Onawunmi, W.-A. Yisak, and E. O. Ogunlana, "Antibacterial constituents in the essential oil of Cymbopogon citratus (DC.) Stapf.," Journal of Ethnopharmacology, vol. 12, no. 3, pp. 279-286, 1984.

[20] C. F. Carson and T. V. Riley, "Antimicrobial activity of the essential oil of Melaleuca alternifolia," Letters in Applied Microbiology, vol. 16, no. 2, pp. 49-55, 1993.

[21] C. F. Carson and T. V. Riley, "Antimicrobial activity of the major components of the essential oil of Melaleuca alternifolia," Journal of Applied Bacteriology, vol. 78, no. 3, pp. 264-269, 1995.

[22] S. G. Griffin, S. G. Wyllie, J. L. Markham, and D. N. Leach, "The role of structure and molecular properties of terpenoids in determining their antimicrobial activity," Flavour and Fragrance Journal, vol. 14, no. 5, pp. 322-332, 1999.

[23] J. E. C. Betoni, R. P. Mantovani, L. N. Barbosa, L. C. di Stasi, and A. Fernandes Jr., "Synergism between plant extract and antimicrobial drugs used on Staphylococcus aureus diseases," Memorias do Instituto Oswaldo Cruz, vol.101, no. 4, pp. 387-390, 2006.

[24] S. Hemaiswarya, A. K. Kruthiventi, and M. Doble, "Synergism between natural products and antibiotics against infectious diseases," Phytomedicine, vol. 15, no. 8, pp. 639-652, 2008.

[25] R. Dryselius, N. Nekhotiaeva, and L. Good, "Antimicrobial synergy between mRNA- and protein-level inhibitors," Journal of Antimicrobial Chemotherapy, vol. 56, no. 1, pp. 97-103, 2005. 

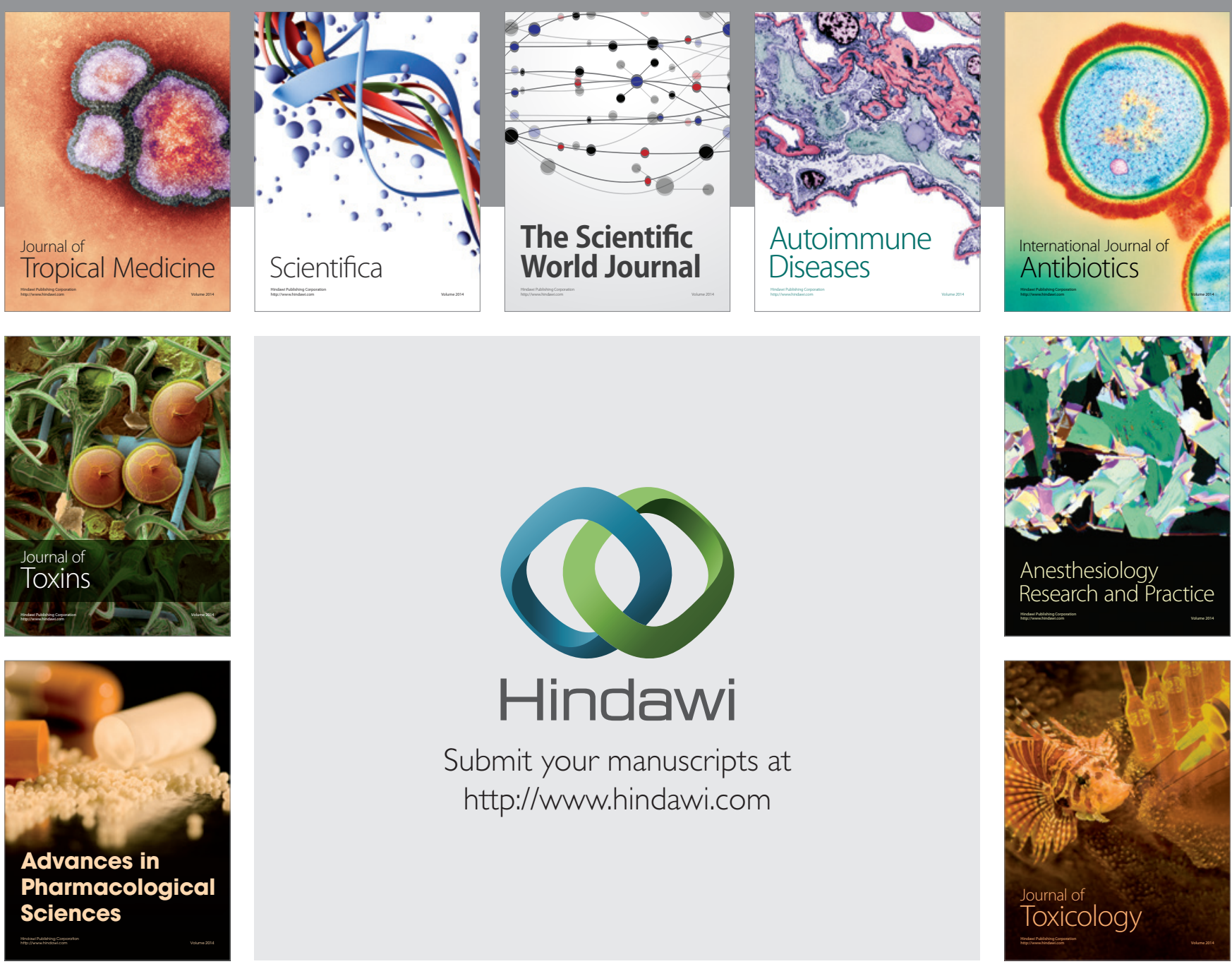

\section{Hindawi}

Submit your manuscripts at

http://www.hindawi.com
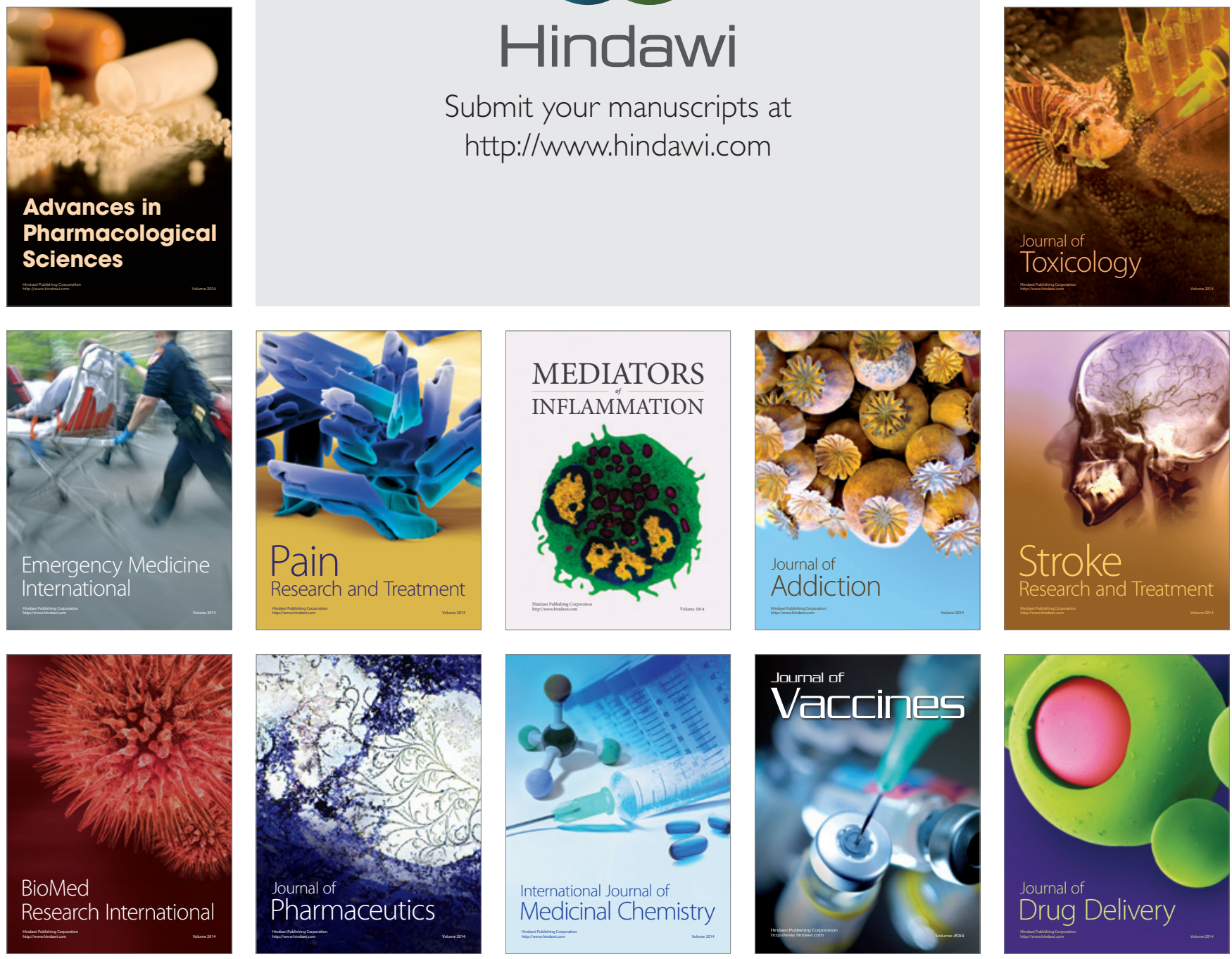